\title{
CONVERGENCE AND DECOMPOSITION FOR TENSOR PRODUCTS OF HILBERT SPACE OPERATORS
}

\author{
C. S. Kubrusly AND P. C. M. VIEIRA
}

\begin{abstract}
It is shown that convergence of sequences of Hilbert space operators is preserved by tensor product and the converse holds in case of convergence to zero under the semigroup assumption. In particular, unlike ordinary product of operators, weak convergence is preserved by tensor product. It is also shown that a tensor product of operators is a unilateral shift if and only if it coincides with a tensor product of a unilateral shift and an isometry. These results lead to a decomposition of a tensor product of contractions into an orthogonal direct sum of tensor products of class $\mathscr{C}_{00}$, strongly stable tensor products, unilateral shift tensor products, and a unitary tensor product.
\end{abstract}

Mathematics subject classification (2000): 47A80, 47A45.

Key words and phrases: Tensor product, Hilbert space operators, convergence, decompositions.

\section{REFERENCES}

[1] A. BRown AND C. PEARCY, Introduction to Operator Theory I - Elements of Functional Analysis, Springer, New York, 1977.

[2] A. BRown AND C. PEARCY, Spectra of tensor products of operators, Proc. Amer. Math. Soc. 17 (1966), $162-166$.

[3] B. P. DUGGAL, Tensor products of operators - strong stability and p-hyponormality, Glasgow Math. J. 42 (2000), 371-381.

[4] P. R. Halmos, Finite-Dimensional Vector Spaces, Van Nostrand, New York, 1958; reprinted: Springer, New York, 1974

[5] C. S. KuBRUSLY, An Introduction to Models and Decompositions in Operator Theory, Birkhäuser, Boston, 1997.

[6] C. S. KubRuSLY, A concise introduction to tensor product, Far East J. Math. Sci. 22 (2006), 137-174.

[7] C. S. KubRUSLY, Tensor product of proper contractions, stable and posinormal operators, Publ. Math. Debrecen 71 (2007), 425-437.

[8] C. S. KUBRUSLY, Regular subspaces of tensor products, to appear.

[9] C. S. Kubrusly, P. C. M. Vieira AND D. O. PinTo, A decomposition for a class of contractions, Adv. Math. Sci. Appl. 6 (1996), 523-530.

[10] M. Reed AND B. Simon, Methods of Modern Mathematical Physics I: Functional Analysis, 2nd edn. Academic Press, New York, 1980.

[11] R. RYAN, Introduction to Tensor Products of Banach Spaces, Springer, London, 2002.

[12] J. StOCHEL, Seminormality of operators from their tensor product, Proc. Amer. Math. Soc. 124 (1996), $135-140$.

[13] B. Sz.-NAGY And C. FolaŞ, Harmonic Analysis of Operators on Hilbert Space, North-Holland, Amsterdam, 1970.

[14] J. WeIDMANN, Linear Operators in Hilbert Spaces, Springer, New York, 1980. 